\title{
Advocating the Development of Next-Generation, Advanced-Design Low-Field Magnetic Resonance Systems
}

\author{
Val M. Runge, MD and Johannes T. Heverhagen, MD, PhD
}

\begin{abstract}
New next-generation low-field magnetic resonance imaging systems (operating in the range of $0.5 \mathrm{~T}$ ) hold great potential for increasing access to clinical diagnosis and needed health care both in developed countries and worldwide. The relevant history concerning the choice of field strength, which resulted in $1.5 \mathrm{~T}$ still dominating today the number of installed systems, is considered, together with design advances possible because of interval developments, since low field was considered for clinical use in the 1980s, and current research. The potential impact of low-cost, advanced-generation low-field magnetic resonance imaging systems, properly designed, is high in terms of further dissemination of health care-across the gamut from industrial to developing countries - regardless of disease entity and anatomic region of involvement, with major niche applications likely as well.
\end{abstract}

Key Words: magnetic resonance imaging, field strength, image quality

(Invest Radiol 2020;55: 747-753)

T his review begins with a consideration of the historical evolution of field strength in the development of clinical magnetic resonance (MR) systems, together with the low-field superconducting systems (operating in the range of $0.5 \mathrm{~T}$ ) existent in the 1980s and 1990s (when these systems were last sold). The main focus of the review then follows, discussing the potential today of low field and the technologic advances ${ }^{1}$ that could be used to maximize the image quality on those systems. It is well time to reconsider low field for clinical imaging. There is a bright future for next-generation, advanced-design systems that offer high image quality, broad flexibility, and a substantial reduction in system cost. This development is likely to greatly expand the use and clinical value of MR, accelerating the dissemination of MR technology worldwide.

\section{THE HISTORICAL EVOLUTION OF FIELD STRENGTH}

There have been remarkably few studies and little comparative research in the transition from $0.014 \mathrm{~T}$ - the operational field strength in 1980 for the first whole-body MR scanner - to $3 \mathrm{~T}$ today, as used for clinical examinations. The original basis for MR imaging (MRI) was first envisioned by Paul Lauterbur at Stony Brook University (United States) and Peter Mansfield at the University of Nottingham (United Kingdom) in the 1970s, with the 2 jointly sharing the Nobel Prize in Physiology/Medicine in 2003. ${ }^{2}$ Lauterbur's fundamental contribution was the use of gradient magnetic fields to obtain spatial information, allowing 2-dimensional (2D) images to be acquired (1973). Mansfield advanced this work further by introducing the concept of frequency and phase encoding using spatial magnetic field gradients, with

Received for publication February 10, 2020; and accepted for publication, after revision, May 22, 2020.

From the Department of Diagnostic, Interventional, and Pediatric Radiology, University Hospital of Bern, Inselspital, University of Bern, Bern, Switzerland.

Conflicts of interest and sources of funding: Partial support was provided by an unrestricted educational grant from Siemens Healthineers.

Correspondence to: Prof. Dr. med. Val M. Runge, MD, Inselspital, Universitätsinstitut für Diagnostische, Interventionelle und Pädiatrische Radiologie, Freiburgstrasse 10 (INO B 106), 3010 Bern, Switzerland.

Copyright (C) 2020 Wolters Kluwer Health, Inc. All rights reserved.

ISSN: 0020-9996/20/5512-0747

DOI: $10.1097 /$ RLI.0000000000000703 the final image able to be reconstructed by the use of a Fourier transform (1977). Shortly thereafter, the team led by Professor Mallard in Aberdeen, Scotland, developed the first whole-body MR scanner. ${ }^{3}$

The first whole-body MR system, that at Aberdeen, was used to scan more than 1000 patients. In August 1980, on this unit, the first clinically useful MR images of a patient were obtained, identifying a primary lung tumor, abnormalities in the liver, and metastatic spread to the bone. By the end of 1982, this machine (Mark I) was already outdated, so rapid were the technological advances in the field, with the unit used only infrequently for small research projects. By that date, the work of the group in Aberdeen was focused on a second prototype, which was to operate at twice the magnetic field of the original system $(0.028 \mathrm{~T})$, still generated though by a resistive magnet.

Diasonics, a company whose MR business was eventually sold to Toshiba, produced the earliest commercial units, standardizing in the early $1980 \mathrm{~s}$ on a field strength $0.35 \mathrm{~T}$ (a 10-fold leap in field strength over the Mark II Aberdeen prototype), using a superconducting magnet. The company vigorously defended their choice of field strength, both in lectures and scientific publications. ${ }^{4}$ Technicare, another early market leader, initially delivered four $0.5 \mathrm{~T}$ clinical units in 1983 (Fig. 1), then standardized on $0.6 \mathrm{~T}$, also strongly defending its choice of field strength. ${ }^{5}$ Siemens' first commercial units, delivered in the mid-1980s, operated at $1.0 \mathrm{~T}$, a field strength chosen and advocated by several other manufacturers at that time. Thus, the magnetic field of choice very rapidly evolved from the initial choice of $0.04 \mathrm{~T}$ to $0.5-1.0 \mathrm{~T}$ (with the evolution as well from resistive to superconducting magnets).

The back cover of the journal Radiology had an advertisement each month for many years in the mid and late 1980s detailing GE's developments in MR and specifically advocating a field strength of $1.5 \mathrm{~T}$. This was a radical idea at the time, with no clinical systems having been delivered with such a high field strength. Major arguments made in this way included statements (some later proven to be false) such as the importance in the future of phosphorus spectroscopy, "which would not be possible at lower field strengths and only achievable at 1.5 T." Largely because of marketing pressure, the other major vendors were driven to invest in the development of clinical 1.5 T units. By the 1990s, delivery of $1.5 \mathrm{~T}$ units dominated the industry.

By the early 2000s, Radiological Society of North America refresher courses were already beginning to debate the pros and cons of $3 \mathrm{~T}$, although clinical experience was almost nonexistent. In general, 3 Twas strongly advocated by speakers and experts in the field during the first part of this decade. ${ }^{6}$ However, in these sessions, comments from the audience and from the few actual users were often mixed. Brain images with 1 major vendor were inferior to those achieved at $1.5 \mathrm{~T}$, because of shading, susceptibility artifacts, and vessel pulsation. Furthermore, in the early part of the decade, only the brain could be imaged with a quality sufficient for clinical examinations. The resulting brain images were superior to those acquired at $1.5 \mathrm{~T}$ only with 1 of the 2 major vendors of the day. And, until short-echo time (TE) 2D gradient echo (GRE) sequences were introduced, ${ }^{8}$ the T1-weighted scans on those units remained inferior to those at $1.5 \mathrm{~T}$.

There were many major challenges in making $3 \mathrm{~T}$ an acceptable scanner for all body regions, ${ }^{8}$ including, for example, longer T1 times, chemical shift (both in plane and through plane), and patient heating (specific absorption rate [SAR] $).{ }^{9}$ One thing noted immediately with 


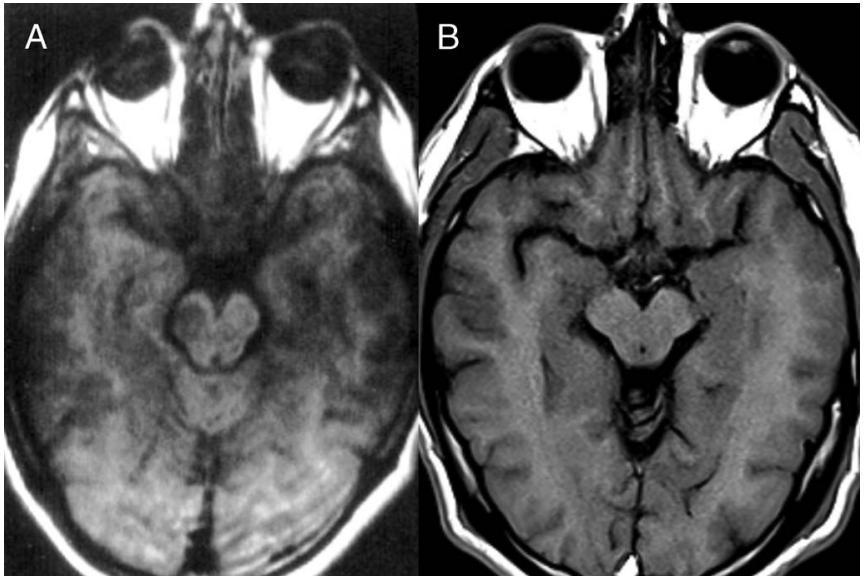

FIGURE 1. Brain imaging, a historical comparison of $0.5 \mathrm{~T}$ in (A) 1984 versus (B) 2020. The scan in 1984 is from a patient with multiple sclerosis (MS), whereas that in 2020 is from a normal volunteer. Scan time was 10 minutes for $(A)$ and slightly more than 4 minutes for $(B)$, with voxel dimensions of $2.2 \times 4.4 \times 10 \mathrm{~mm}^{3}$ versus $1 \times 0.7 \times 5 \mathrm{~mm}^{3}$. A large, chronic, right pontine MS plaque is noted in (A), with the scan otherwise nondiagnostic due to poor SNR and CNR. Note the marked improvement in overall image quality (including specifically SNR and spatial resolution) from 1984 to 2020, with both images acquired at low field, owing to interval technologic advances.

early $3 \mathrm{~T}$ brain scans was the difficulty of the patient to hold sufficiently still to obtain the achievable anatomic resolution without substantial motion artifacts. Brain images could be acquired in a clinically acceptable scan time ( 5 minutes) with very high in-plane spatial resolution (eg, $0.5 \times 0.5 \mathrm{~mm}^{2}$ ), but many of the resultant scans were of poor image quality because of patient motion. However, the scans in which the patient did not move were of exceptional quality owing to the spatial resolution that could be achieved. Major adjustments in scan parameters, and indeed in the scan sequences, that were used for image acquisition were required. Even today, the debate continues in some anatomic areas as to whether $1.5 \mathrm{~T}$ is superior, for example, in cardiac imaging and with metal implants. The quality of spine images at $3 \mathrm{~T}$ slowly improved during the 2000s, requiring redesign of the spine coils and sequences more amenable to use at $3 \mathrm{~T}$. In other clinically important areas, such as timeof-flight magnetic resonance angiography (MRA), contrast-enhanced MRA, and susceptibility imaging, 3 T was widely recognized very early as being the field strength of choice. By the end of the 2000s, 3 T was recognized as superior to $1.5 \mathrm{~T}$, when images were acquired with appropriate protocols, for the brain, head and neck, spine, and musculoskeletal system. Regardless of these advantages, even today, of every 3 clinical MR units sold, only 1 is $3 \mathrm{~T}$ and the other 2 are $1.5 \mathrm{~T}$-with this largely because of cost. For much of the history of MR, system costs were in the range of US\$1 million per tesla, although these have fallen in recent years. There is also considerable variability in the purchase price depending upon how a system is equipped, with installation costs adding hundreds of thousands of dollars.

\section{LOW-FIELD IMAGING IN THE 1980s AND 1990s, CONTRASTED WITH THE DEVELOPMENT OF 1.5 T}

Early scientific publications advocating high-field-strength imaging often ignored the possibility of low-field imaging. ${ }^{3}$ One of the major manufacturers, being a late entrant into the field, heavily emphasized the advantages of $1.5 \mathrm{~T}$ - the field strength the company had chosen to pursue as a marketing strategy, pushing the entire industry in this direction. The development of $1.5 \mathrm{~T}$ imaging continued forward at breakneck speed, despite prominent evidence of little, if any, advantage in terms of medical diagnosis and sensitivity to disease regarding major areas of clinical utilization. In a large-scale trial involving 132 patients with suspected multiple sclerosis, no difference in accuracy, sensitivity, or specificity was noted between scans conducted at 0.5 and $1.5 \mathrm{~T} .{ }^{10} \mathrm{In}$ this study, imaging parameters were identical, with the exception of bandwidth optimization for low field strength. Turning to the musculoskeletal system, 114 patients referred for imaging of the knee were evaluated at both 0.5 and $1.5 \mathrm{~T}$ with identical sequences, again with bandwidth optimization and also a slightly longer imaging time at the lower field strength. ${ }^{11}$ No advantage for higher field strength was found regarding accuracy and diagnosis of anterior or posterior cruciate ligament and meniscal tears. It is pertinent to remember that the arguments favoring high magnetic field, $1.5 \mathrm{~T}$ and above, included prominentlyin addition to high signal-to-noise ratio (SNR) (and thus the potential for faster imaging and higher spatial resolution) - spectroscopy and functional MRI, which are rarely used today clinically.

In comparing 0.5 and $1.5 \mathrm{~T}$, the hypothesis still stands today as it did in 1996 "that applications that require very fast imaging, very high resolution imaging, or detection of very small image intensity changes may demonstrate diagnostic advantages for high magnetic field." 12 However, such applications constitute only a small part of the clinical population that is routinely imaged. Using receiver operating characteristic analysis, the same group in Canada demonstrated that for the 2 common diagnostic tests (multiple sclerosis, knee) previously discussed, $0.5 \mathrm{~T}$ MRI was diagnostically equivalent to $1.5 \mathrm{~T}^{12}$ This held true despite the higher SNR and contrast-to-noise ratio (CNR) at $1.5 \mathrm{~T}$ in their comparison. Important in this consideration, however, is bandwidth optimization. For human imaging, SNR is a linear function of field strength, assuming that receiver bandwidth is held constant and the repetition time (TR) is long compared with T1 (which prolongs with higher field strength). But if bandwidth is allowed to vary with field strength, so that chemical shift is always a constant pixel shift, then SNR scales with the square root of field strength. The CNR is a more complicated situation, owing to T1 increasing with field strength. For T1-weighted scans, the advantage for $1.5 \mathrm{~T}$ over $0.5 \mathrm{~T}$ in CNR can be less than $20 \%$. Alternatively, for scan sequences with very little T1 contribution, there can be a $40 \%$ difference in CNR between 0.5 and $1.5 \mathrm{~T}$.

A question that remains to be answered in comparing 0.5 and $1.5 \mathrm{~T}$, unaddressed by previous research, is the performance of timeof-flight MRA and contrast-enhanced MRA on next-generation, advanced low-field systems (Fig. 2). These scan techniques were developed relatively late in the evolution of MR, and specifically on $1.5 \mathrm{~T}$ systems. ${ }^{13}$ Whether advances in technology and scan acquisition techniques will close the gap in image quality for MRA, if indeed such a gap is significant, between low and high field is yet to be seen.

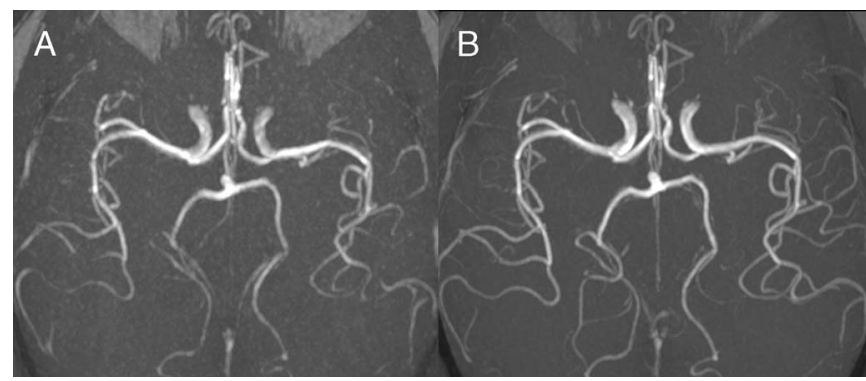

FIGURE 2. Time-of-flight MRA, which was developed for clinical use at $1.5 \mathrm{~T}$ relatively late in the history of $\mathrm{MR}$, performs well at $0.55 \mathrm{~T}$. Thick axial maximum intensity projection reformats are presented from multislab 3D acquisitions at (A) $0.55 \mathrm{~T}$ and (B) $1.5 \mathrm{~T}$ in the same normal volunteer. Voxel dimensions were $0.5 \times 0.5 \times 0.5 \mathrm{~mm}^{3}$ in each instance, with approximate scan times of 6 versus 5 minutes. The more distal arterial branches are not depicted as well at $0.55 \mathrm{~T}$ because of the lower SNR (and CNR) at this field strength. 


\section{ADDRESSING LOW-FIELD MR TODAY}

The question arises as to what new opportunities exist for making MR accessible to a broader patient population, ${ }^{14}$ or simply just more cost efficient. ${ }^{15}$ The development of high field was motivated by the promise of increased SNR and, thus, spatial resolution (in the end, image quality). However, for example, the march from $0.5 \mathrm{~T}$ to $1.5 \mathrm{~T}$ to $3 \mathrm{~T}$, such as in spine imaging, only led to a change in slice thickness for routine scans from $5 \mathrm{~mm}$ to $4 \mathrm{~mm}$ to $3 \mathrm{~mm}$. Moreover, the discussion regarding image quality at 1.5 versus $3 \mathrm{~T}$ in spine imaging still continues, owing to image degradation at $3 \mathrm{~T}$ on the basis of CSF motion and chemical shift issues (both through and in plane). For certain clinical questions, slice thickness - as given in the example comparing the utility of the 3 field strengths - can be critical. Thus, a low-field system would need to be able to achieve this (ie, have sufficient SNR), or the applications demanding thin section imaging should be sufficiently small in number to allow justification of purchase of a system without such capabilities (Fig. 3).

Along the way, in the development of 1.5 and $3 \mathrm{~T}$, additional research was required to navigate the new problems raised by high field, for example, in routine T1-weighted imaging, owing to the prolonged tissue T1s and with GRE (as well as echoplanar) techniques due to increased tissue susceptibility. Higher fields also led to increased SAR and - noted previously — cost, together with greater concern regarding patient safety. Today, 1.5 and $3 \mathrm{~T}$ are seen as clinical standards, with the first clinical $7 \mathrm{~T}$ systems available in 2017, while imaging at low field $(0.5-0.6 \mathrm{~T})$ has largely disappeared. The specifications and infrastructure requirements of MR systems today have also grown substantially and made MR inherently one of the most expensive modalities in radiology, limiting patient access and utilization.

In light of the economic pressure on health care systems worldwide, it seems worth revisiting as well the following questions: is it possible to reduce the cost of the most expensive part of an MR system, the magnet, while at the same time providing excellent image quality, possibly adding new diagnostic value (eg, with interventional MR), and making MR more accessible (in several meanings of the word)? Is there a missed opportunity in the journey that clinical MR and the research community have taken in the last 2 decades?

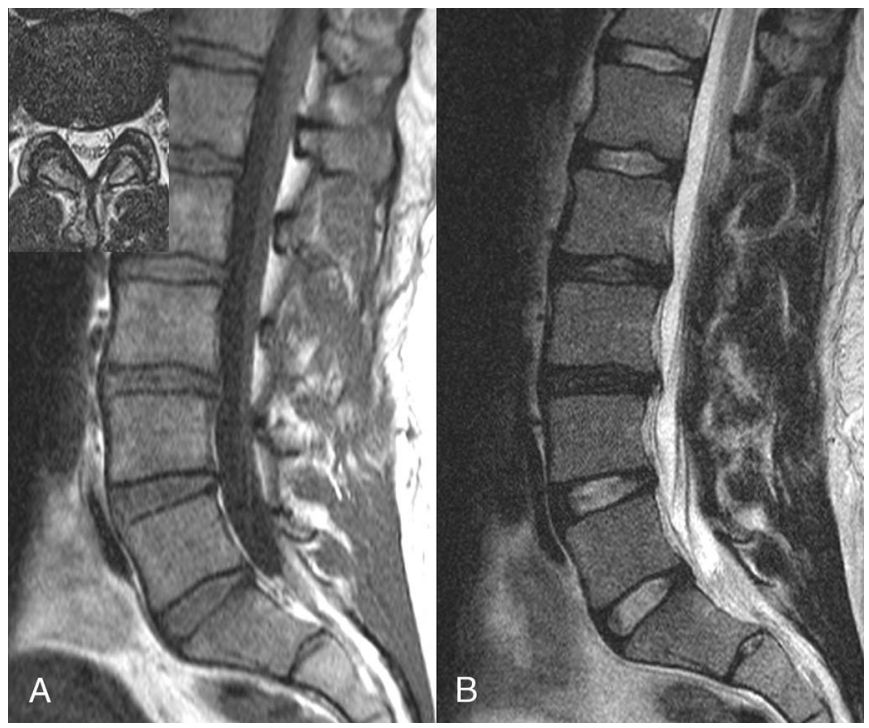

FIGURE 3. Sagittal (A) T1- and (B) T2-weighted 2D fast spin echo scans at $0.55 \mathrm{~T}$ of the lumbar spine, with (inset) the axial T2-weighted scan also presented. The slice thickness in each instance was $4 \mathrm{~mm}$. Scan times were 4 minutes, 4 minutes 31 seconds, and 3 minutes 24 seconds. There is disk dessication at $\mathrm{L} 2-3$ and $\mathrm{L} 3-4$, with a small right paracentral annular tear seen on the T2-weighted scans at L3-4.
Intrinsic advantages for low-field systems based on the science of MR include shorter tissue T1 and longer T2* (allowing more time-efficient sequence acquisitions), reduced susceptibility effects, and reduced SAR (tissue heating). The latter also leads to diminished heating of metal devices and implants and lessens pulse sequence parameter constraints (eg, allowing larger tip angles and/or greater numbers of slices). When low-field technology was last explored in the 1980s, spiral acquisition, parallel imaging, and iterative reconstruction, as well as many other current acquisition and postprocessing strategies, were not yet invented. A new look at low-field technology today has the potential to lead to advanced, next-generation low-field MR systems with substantially lower cost, flexible utilization, and excellent image quality. If development of $0.5 \mathrm{~T} \mathrm{MR}$ is fully explored, this could lead to a wide range of new scanners, extending from basic systems destined for a remote area or developing nations to high-end niche systems including dedicated interventional, intraoperative, and emergency room installations.

\section{MAGNET AND RECEIVER COIL TECHNOLOGY}

Bore size is an important consideration in the design of an advanced, next-generation low-field system. Traditionally, superconducting MR magnets had a $60-\mathrm{cm}$ bore, although the original Diasonics $0.35 \mathrm{~T}$ scanner (as well as an early $1.5 \mathrm{~T}$ GE system) had a $55-\mathrm{cm}$ bore. ${ }^{6}$ Specialty units, with reduced bore size, have also been developed, such as the Siemens Allegra - a $3 \mathrm{~T}$ head only scanner introduced in the early 2000 s (still in use today) ${ }^{16}$ - and more recently the Synaptive Medical Evry - an innovative, new $0.5 \mathrm{~T}$ dedicated head unit with approval now in Canada in 2020. ${ }^{17}$

The Magnetom Espree represented at its launch in 2004 a highly innovative whole-body MR design, with the first major increase in bore size, to $70 \mathrm{~cm}$, combined with an ultrashort system length of $125 \mathrm{~cm}$. This unit went on to dominate the industry largely because of patient comfort and the increasing weight (and thus girth) of patients in the developed world. The comparatively small amount of superconducting wire needed for a low-field system (when compared with higher-field systems) could potentially allow a cost-efficient design for an ultrawide-bore system, with bore dimensions theoretically possible in the range of 80 to $90 \mathrm{~cm}$. Along with bore size, an appropriate choice of length of the MR unit would be important. An alternative approach, possible in the more distant future, is the development of high-temperature superconducting magnets for clinical MR.

Siting logistics are also a huge barrier to increasing the adoption of MRI in many settings, including operating rooms, remote clinical sites, and developing world clinics. Cryogen free or zero boiloff magnets, with elimination of the quench pipe and small exclusion zones, offer a very promising direction for the immediate future.

Substantially more affordable and accessible imaging could be the result, ushering in for the current decade potentially increased patient comfort, improved bore access, lower system weight, combined with lower cost and thus greater accessibility to MR diagnosis worldwide. It should also be kept in mind that there have also been major advances since the 1980s in gradient coil and receiver coil technology that could be applied to a next-generation low-field system. For example, in the 1980s, receiver coils were far from optimized, with head coils both larger in diameter and longer than needed, with radiofrequency (RF) reception for body imaging provided by a coil adjacent to the body transmit coil (far away from the patient), and multichannel/flexible coils still decades away from development.

\section{GRADIENT PERFORMANCE FOR ROUTINE CLINICAL APPLICATIONS}

After the magnet, the gradient system is the second largest cost component of an MRI system. As with field strength, gradient performance has continued to increase over the past decades with (almost) every new generation of MRI systems. Although the slew rate of gradients 
for whole-body systems already reached the physiological limits of stimulation a few years ago, higher and higher gradient amplitudes are being brought to the market, allowing for research applications like diffusion tensor imaging with very high resolution. On the downside, manufacturing costs, complexity, and raised requirements regarding connection values, increasing power consumption, and cooling requirements are to be noted.

Somewhat analogous to modern (standard) cars, which easily reach top speeds of 200 kilometers an hour but are restricted by speed limits to substantially below this, it is worth revisiting when and how often peak amplitudes in gradients are actually used and really required in daily clinical routine. Ninety-five percent of daily clinical use cases encompass routine brain imaging, spine imaging, musculoskeletal imaging (Fig. 4), and abdominal-pelvic imaging (Fig. 5). If one accepts to exclude some advanced use cases like diffusion tensor imaging, functional MRI, and cardiac imaging, diffusion-weighted imaging (DWI) in oncology and neurology is certainly the most demanding application when it comes to gradient performance. The highest acquired $b$ value in typical neuro and body protocols is commonly in the range of $b=800$ to $1000 \mathrm{~s} / \mathrm{mm}^{2}$.

To achieve a certain $b$ value, duration, interval between, and amplitude of gradients are the accessible factors at a given field strength. Although the $b$ value only scales with the square of the gradient amplitude, it scales with (about) the third power of the gradient duration. Accordingly, having a substantially lower maximum gradient amplitude will only result in a slightly longer duration of the gradient pulse to achieve the same $b$ value. More concretely, with a slew rate of $40 \mathrm{~T} / \mathrm{m} /$ $\mathrm{s}$ and an amplitude of $25 \mathrm{mT} / \mathrm{m}$, TEs of a standard single-shot DWI protocol of the brain will be in the range of 10 to 15 milliseconds longer than with a common high-end gradient system.

Being used to imaging at higher field strength, this actually may seem to be quite a substantial increase in TE. At low-field, however, boundary conditions for DWI are different compared with high field, and the balance between parameters like T2*, T2, TE, SNR, and echo spacings is substantially changed (Fig. 6). In DWI at higher fields, we all have been trained to make TE and echo spacing as short as possible to minimize susceptibly artifacts and to maximize SNR. T2* decay and

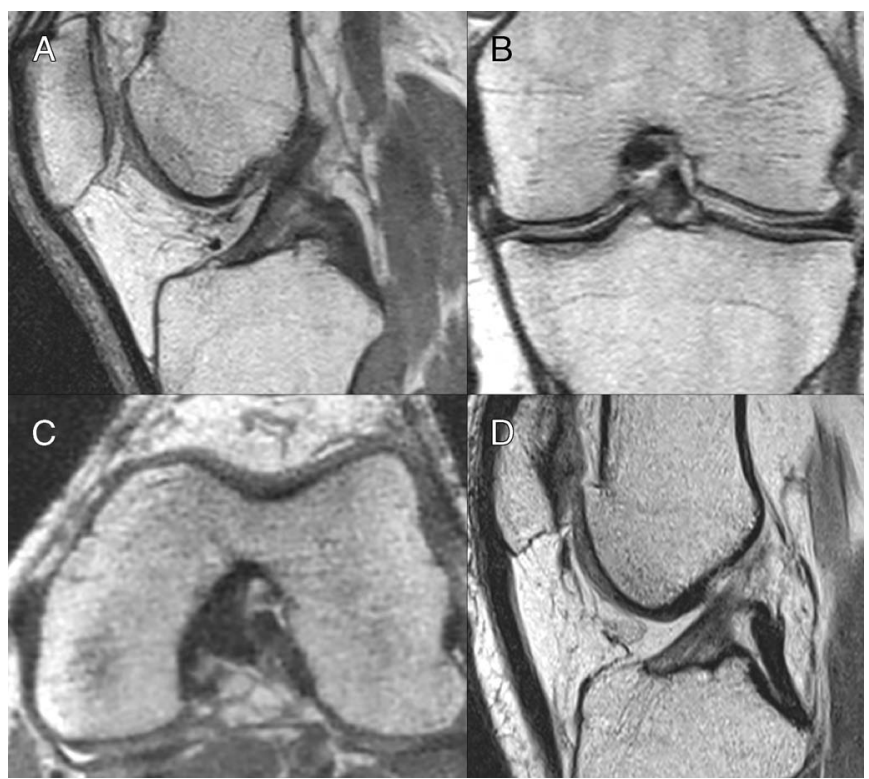

FIGURE 4. A-C, Sagittal, coronal, and axial reformatted images from a 3D proton density weighted SPACE knee acquisition at $0.55 \mathrm{~T}$. Scan time was 4 minutes 9 seconds, with a slice thickness of $0.7 \mathrm{~mm}$. Also presented, in (D), is a 3-mm, high in-plane spatial resolution $\left(0.26 \times 0.21 \mathrm{~mm}^{2}\right)$, midline 2D proton density weighted sagittal image (in the same normal volunteer) from a 5 minute 2 second fast spin echo acquisition at 0.55 $\mathrm{T}$-further depicting the anterior and posterior cruciate ligaments.
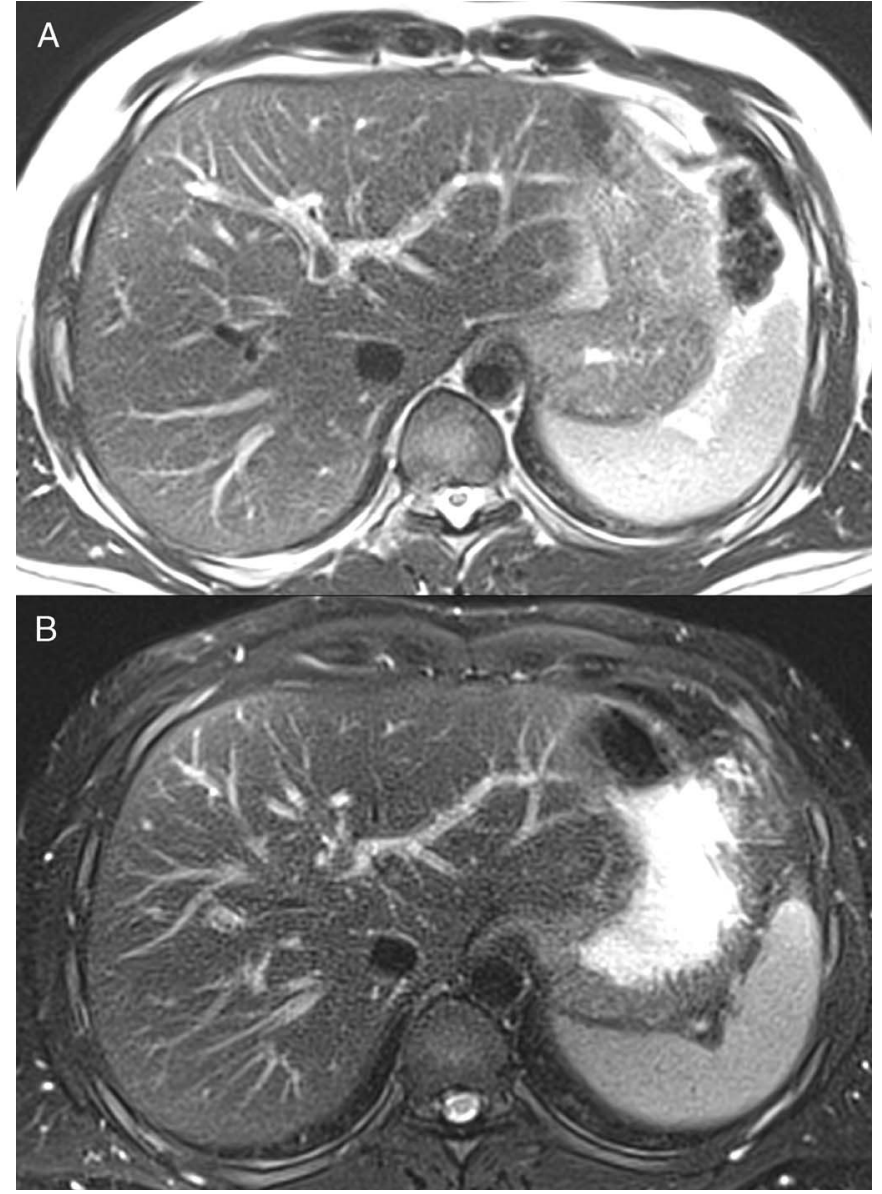

FIGURE 5. Axial respiratory triggered 2D T2-weighted images of the liver, in a normal volunteer, obtained at (A) $0.55 \mathrm{~T}$ and (B) $1.5 \mathrm{~T}$ using a motion insensitive, multi-shot turbo spin echo sequence. The slice thickness was $6 \mathrm{~mm}$ in each case. The TR was prolonged to 5300 milliseconds at $0.55 \mathrm{~T}$ as compared with 3600 milliseconds at $1.5 \mathrm{~T}$. Parallel imaging was used in each instance, but with a factor of 2 at the lower field strength as compared with 3 at the higher field strength. The scan time was 4 minutes 30 seconds at $0.55 \mathrm{~T}$ versus 1 minute 48 seconds at $1.5 \mathrm{~T}$.

susceptibility are less of an issue at lower fields, and thus, longer echo spacing is acceptable. The SNR issue with longer TEs can be compensated with lower readout bandwidths.

As illustrated with the example of DWI, the balancing of imaging parameters is an optimization problem with different boundary conditions at lower fields, which requires us to think outside the box. The images in this publication are acquired with the above-mentioned gradient settings. By careful design and implementation, the potential disadvantages of a nontraditionally designed gradient system can be mitigated, with resultant high image quality. Designing for lower cost in the gradient system, as with the magnet itself, offers substantial benefits in terms of broadening access to routine MRI.

\section{IMAGE CONTRAST}

$\mathrm{T} 1, \mathrm{~T} 2$, and $\mathrm{T} 2 *$ change, with variability between tissues, at low field when compared with 1.5 T. This offers both advantages and potential disadvantages (Fig. 7). T1 is on average shorter by a third (advantageous for T1-weighted scans), T2 longer by a fourth, and T2* longer by almost half (advantageous for both echo-planar and spiral acquisitions). The lung is unique, where $\mathrm{T} 2 *$ is more than 3 times as long. ${ }^{18}$ 


\section{SPIRAL IMAGING (IMAGING EFFICIENCY)}

The prolonged T2* at low field can be exploited for improved signal sampling efficiency. This proves to be nearly double for both balanced steady-state free precession and spin echo techniques with a spiral out acquisition (when compared with a typical Cartesian acquisition). ${ }^{19}$ The end result is an increase in SNR, which varies both by tissue and technique (GRE vs spin echo) but in each instance is near double. ${ }^{18}$ By theory, SNR scales linearly with magnetic field strength, although the actual achieved SNR improvement is often lower. Thus, $1.5 \mathrm{~T}$ could offer potentially 3 times the SNR of $0.5 \mathrm{~T}$ (the rationale for high field imaging). Yet, as known today, the gain in SNR with field strength could be largely counterbalanced by a 2 times SNR gain at 0.5 T owing to the implementation of spiral out acquisition strategies.

\section{SIMULTANEOUS MULTISLICE}

As at higher field strengths, the simultaneous multislice (SMS) technique may find applicability in low-field imaging. The SMS technique has been used at 1.5 and $3 \mathrm{~T}$ to reduce scan time and/or increase the number of acquired slices. ${ }^{20}$ The technique has been applied in diffusion-weighted, single-shot echoplanar imaging by reducing the TR, and with fast spin echo scans (for proton density and T1 and T2 weighting) to provide greater anatomic coverage. At low field, SMS could and likely will be used in a different way, to improve SNR while maintaining scan time.

\section{ITERATIVE DENOISING}

Iterative denoising of MRI data holds great promise for improved image quality for scan acquisitions with borderline SNR, for example, when resolution is increased or acquisition time is decreased. ${ }^{21,22}$ This approach thus has particular importance for improving image quality on low-field scanners, where SNR is lower than at 1.5 or $3 \mathrm{~T}$. One limitation to the application of iterative denoising is the potential to render lesions less conspicuous, for example, by the introduction of image blur. Methods for iterative denoising must necessarily avoid blurring effects and specifically not introduce image artifacts. ${ }^{22,23}$

Spatial varying noise limits both spatial resolution and acquisition speed with the application of multichannel coils for signal reception,

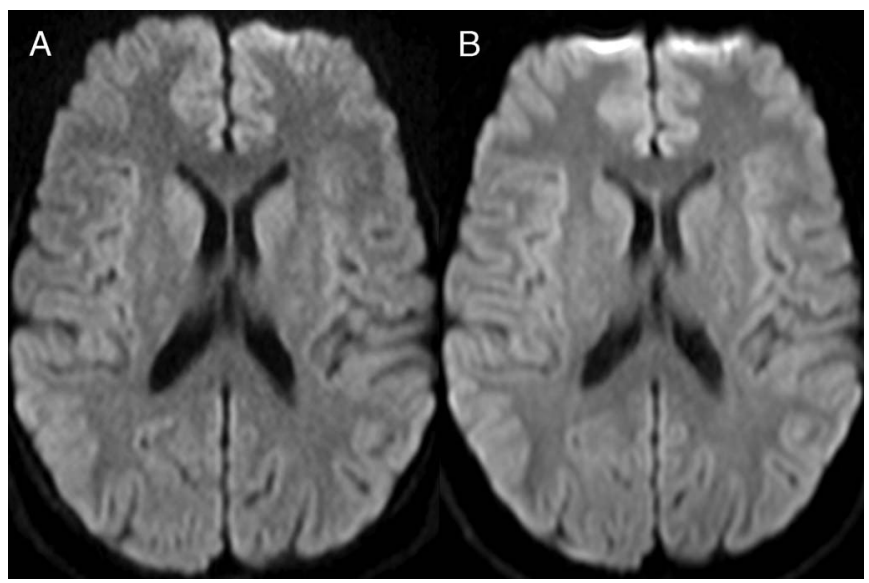

FIGURE 6. A comparison of 2D single-shot diffusion-weighted echoplanar imaging (trace weighted images, $B=1000$ ) at $(A) 0.55 \mathrm{~T}$ and (B) 1.5 T. The slice thickness was $5 \mathrm{~mm}$ and pixel dimensions were identical. Note the mild, characteristic, image imperfections at $1.5 \mathrm{~T}$, including the image distortion anteriorly on the $1.5 \mathrm{~T}$ image (caused by susceptibility effects from the frontal sinus), the mild image blurring (seen well in comparison to the $0.55 \mathrm{~T}$ image), as well as the slight image foreshortening. The scan times were 3 minutes 10 seconds versus 1 minute 38 seconds with the difference largely caused by the doubling of signal averages for the acquisition at $0.55 \mathrm{~T}$ as compared with $1.5 \mathrm{~T}$.

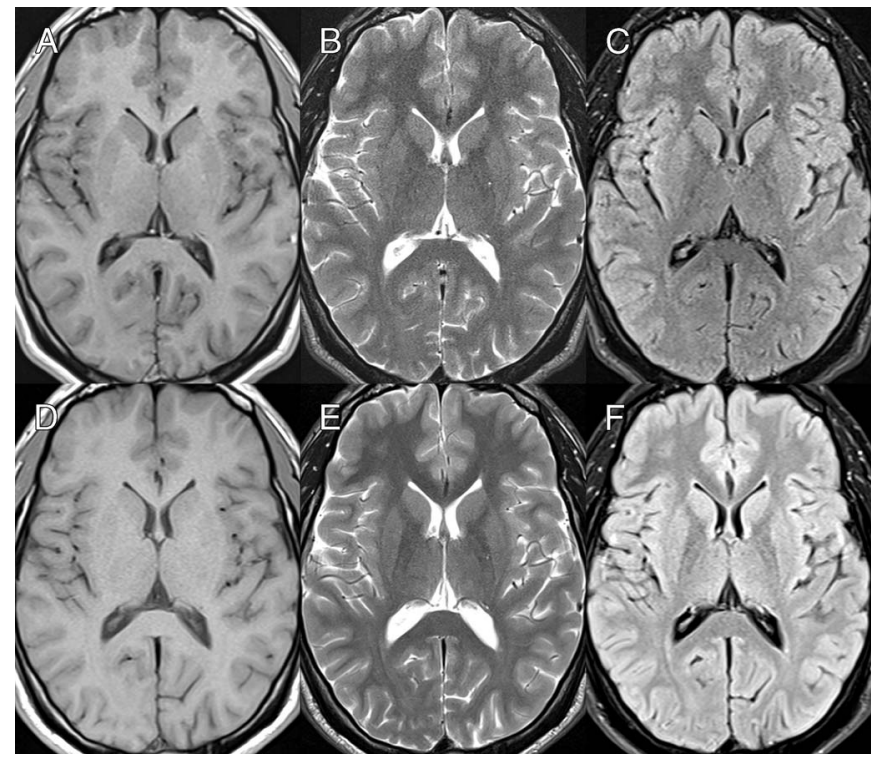

FIGURE 7. A comparison of 2D fast spin echo (A, D) T1-weighted, (B, E) T2-weighted, and (C, F) fluid-attenuated inversion recovery (FLAIR) images of the brain obtained at (A-C) $0.55 \mathrm{~T}$ and (D-F) $1.5 \mathrm{~T}$ in a normal volunteer. The slightly lower SNR at $0.55 \mathrm{~T}$ is evident for all scan techniques. Also noted is the slightly improved T1 contrast at $0.55 \mathrm{~T}$ due to the prolongation of $\mathrm{T} 1$ with increasing field strength. The slice thickness was $5 \mathrm{~mm}$ in all instances, with in-plane spatial resolution comparable for each field strength comparison. Scan times were $(A, D)$ 4 minutes 16 seconds versus 1 minute 51 seconds, $(B, E) 3$ minutes 48 seconds versus 3 minutes 15 seconds, and (C, F) 4 minutes 30 seconds versus 4 minutes 12 seconds.

with iterative denoising able to be applied to almost all routine $2 \mathrm{D}$ and 3D MRI acquisitions. Complex-valued image data from scan acquisition is exported after parallel image reconstruction and coil combination, but before interpolation and magnitude reconstruction. Also exported at the same time is supplementary, critical image information, specifically regarding image normalization, k-space filtering, and noise calibration. The image data are then iteratively denoised by thresholding using orthogonal wavelet transforms, with the thresholds spatially adapted to the local noise level. After denoising, the complex-valued image data are reimported to the standard scanner reconstruction pipeline and magnitude images are calculated. No manual tuning of filter parameters is needed, with the algorithm automatically adapting to changes in acquisition/reconstruction settings and scan conditions. In comparison with conventional noise suppression filters (used routinely by all MR manufacturers), better results are achieved, owing both to the exploitation of supplementary information about the acquisition, reconstruction, and noise and by working with 3D complex-valued data. Application of iterative denoising is anticipated to enable on the order of a $25 \% \mathrm{SNR}$ gain or, alternatively, a $30 \%$ reduction in scan time.

\section{DEEP LEARNING RECONSTRUCTION}

Another highly promising development, still in its infancy, is to utilize deep neural networks either in the direct transformation of raw data into images or to optimize the quality of otherwise nondiagnostic images. For illustration purposes, take the simple example of a fast, low-resolution scan acquired at low field. If a neural network is trained with high-resolution images from the same field strength or even with images in the same subject acquired at a higher field strength, the network will establish "neural connections" to associate certain features in the low-quality image with features in the high-quality image. After training on a few thousand images, the network can apply its "knowledge" and improve the resolution of images, a process frequently called 
super-resolution processing. Beyond such image optimization strategies (image-to-image-network), deep learning may also be beneficial to mitigate highly unpredictable artifact patterns, such as streaking in radial imaging ( $\mathrm{k}$-space-to-image).

\section{IMAGING IN HIGH-SUSCEPTIBILITY REGIONS}

Susceptibility is a measure of the extent to which a substance is magnetized when exposed to an external magnetic field. Tissues exhibit a range of susceptibility, interfering with image quality on MR in areas where there is a large change in susceptibility. Both geometric distortion and signal pile up (artifactual areas of high and low signal intensity) result (Fig. 8). Susceptibility is proportional to magnetic field strength and is thus substantially lower at low field compared with high field. Common areas where a susceptibility effect is seen in clinical MRI, and can interfere with clinical diagnosis, include air-tissue interfaces, metal implants, and trabecular bone. The anatomic regions most affected include the sella, internal auditory canal, optic canal, paranasal sinuses, skull base, oral cavity, pharynx, larynx, esophagus and airways, lungs, and bowel. A marked improvement in image quality can be expected in these areas at low field, considering the factor of susceptibility by itself, together with the imaging of metal within the body. This may finally open the field of lung imaging for MR, which theoretically offers high clinical value because of its soft tissue contrast. Low-field MR could offer markedly improved imaging of parenchymal lung disease, for example, cystic fibrosis, as has been shown in early clinical images from an advanced-generation, high-performance $0.55 \mathrm{~T}$ system. ${ }^{18}$ One major advantage to lung imaging with MR, particularly in the pediatric population but as well in any chronic disease requiring repeated imaging, would be the elimination of x-ray radiation - specifically the high radiation dose over the patient's lifetime due to repeated CT examinations.

\section{ACOUSTIC NOISE}

Theory predicts and experimental results have confirmed that doubling of the magnetic field produces an increase of $6 \mathrm{~dB}(\mathrm{~L})$ in acoustic noise power generated by the gradient coils. ${ }^{24}$ It should be noted that $\mathrm{dB}$ is a logarithmic scale. Normal conversation is at about $60 \mathrm{~dB}$, a vacuum cleaner is $75 \mathrm{~dB}$, sounds above $85 \mathrm{~dB}$ are considered harmful, and a subway is 90 to $95 \mathrm{~dB}$. Evaluation of a clinical scanner and gradient system in 2002 revealed acoustic noise to range from $77 \mathrm{~dB}$ at $0.5 \mathrm{~T}$ (with the lowest noise scan) to $98 \mathrm{~dB}$ at $1.5 \mathrm{~T}$ (with the highest noise sequence). With all else equal, and choosing a midrange

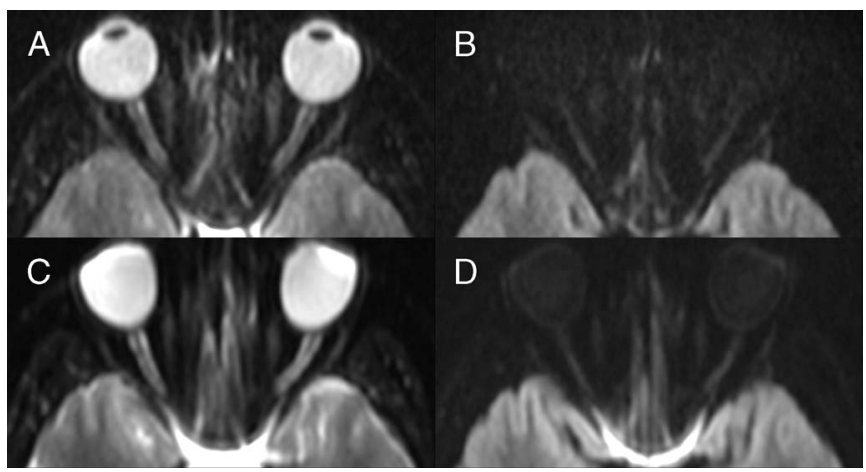

FIGURE 8. ( $A, C) B=0$ and $(B, D) B=1000$ diffusion-weighted scans are presented from a normal volunteer imaged at $(A, B) 0.55 \mathrm{~T}$ and $(C, D) 1.5 \mathrm{~T}$ with single-shot echoplanar technique. Note the marked distortion of the globes, poor visualization of the optic nerves, and prominent susceptibility artifact from the sphenoid sinus (which lies immediately below the depicted imaging plane) at $1.5 \mathrm{~T}$, a reflection of the increase in magnetic susceptibility with field strength. Pulse sequence parameters were similar between field strengths (the same as in Fig. 6), other than signal averages ( 8 vs 4 at 0.55 vs $1.5 \mathrm{~T}$ ). level of sound, changing from a 1.5 to a $0.5 \mathrm{~T}$ MR will reduce the noise of the gradients from that of a subway to that of a door bell.

\section{CONTRAST MEDIA}

A question that does arise for low-field MR is the efficacy of contrast media, and specifically the gadolinium chelates within this range of field strengths. The short answer is that this is not an issue of concern. There are abundant data concerning the T1 relaxivity of gadolinium chelates at lower field strengths, published in the early years of development of clinical MR. Indeed, the clinical trials required for regulatory approval of the gadolinium chelates were largely performed including low-field systems.

A brief history of the early development of contrast media for MR is included in the 2 paragraphs that follow, focusing on data obtained during that time at fields strengths below $1 \mathrm{~T}$. Shown at the 1984 American Society of Neuroradiology meeting ${ }^{25}$ were the first images demonstrating the efficacy of a gadolinium chelate (the class of contrast media used today for MR), these being acquired at $0.5 \mathrm{~T}$. Also established was the efficacy of the agent across a broad range of field strengths, from $0.15 \mathrm{~T}$ on the low end to $1.5 \mathrm{~T}$ on the high end.

The approval of the first agent in the United States, gadopentetate dimeglumine (Magnevist), used in the research previously described, was based on phase II studies that were conducted at 4 early MR sites-Dallas, Huntington Beach, Nashville, and San Francisco. Three of the MR units operated at a field strength of $0.35 \mathrm{~T}$, and 1 at a field strength of $0.5 \mathrm{~T}^{26}$ The approval of Gd HP-DO3A (ProHance) followed, as the second MR contrast agent in the United States and the first macrocyclic agent, with patients studied on scanners ranging in field strength from 0.2 to $1.5 \mathrm{~T}^{27}$ That the agents are effective across a wide range of field strengths was demonstrated in these early studies, confirming that the use of gadolinium chelates will not be an issue in the introduction of new, advanced-design low-field MR scanners. Dedicated in vitro relaxivity studies also support this conclusion, with no statistically significant difference noted in $\mathrm{T} 1$ relaxivity between 0.5 and $1.5 \mathrm{~T}$ in the 2005 study by Rohrer et $\mathrm{al}^{28}$ and in a second study published in 2015 that included 3 and $7 \mathrm{~T}^{29}$ Indeed, in the former study, there was a tendency toward higher relaxivity (specifically $\mathrm{T} 1$ contrast effect) at $0.5 \mathrm{~T}$ for the gadolinium chelates, as confirmed in 2006 by proton relaxometry. ${ }^{30}$ This is, regardless, counteracted by the prolongation of T1 with increasing field strength. ${ }^{31}$

\section{INTERVENTIONAL MR}

RF heating is a potential concern with MR, and in particular with interventional MR and catheterization. Many factors influence heating, although it is dominated by (scaling with) the operating field strength (and more specifically the corresponding Larmor frequency). Low field thus offers a major advantage over high and ultrahigh fields. Many of the currently available cardiac catheterization devices were recently identified as being candidates for use at low field, owing to the observation of less than $1^{\circ} \mathrm{C}$ of heating during 2 minutes of continuous imaging. ${ }^{18}$ Improved bore access would also be markedly advantageous for an interventional system, which could be provided by a further increase in bore dimensions over the current $70 \mathrm{~cm}$ open-bore systems.

The lower price of a low-field system (including the system itself, installation, and service/cryogens) would make more practical dedicated installations for interventional work in radiology and cardiology. Patient monitoring would be eased by the lower field strength, owing to fewer problems caused by the interaction of the magnetic field with monitoring equipment, reduced magnetohydrodynamic effects (induction of currents in flowing blood, eg, in the aorta), and the smaller/ closer 5 gauss line. Larger-bore dimension and lower magnetic field together would enlarge the patient group in which an interventional procedure could be performed, likely making possible as well interventions in the most difficult patients, who need better monitoring, and 
potentially including patients with active device implants (such as pacemakers). Although a possible niche application of an advanced generation $0.5 \mathrm{~T}$ system, the advantages of such an installation might revolutionize interventional MR, leading to dedicated systems in many departments similar to what has evolved with CT-guided procedures. ${ }^{32}$

A magnetic field lower than 1.5 Twould result, importantly, in a reduction of susceptibility artifacts, with less artifacts from catheters and needles. True fast imaging with steady-state free precession (FISP), which is the work horse, pulse sequence wise, for interventional work (used for image guidance) also performs better at lower fields. Specific absorption rate limits will not be a constraint, as opposed to at $1.5 \mathrm{~T}$. The SNR loss can be countered by more efficient sampling and flip angle adjustments. Bending artifacts will be less. For thermometry, adapted sampling strategies (again taking advantage of the longer T2*) will likely provide sufficient temperature to noise level and acquisition speed. Overall, from every perspective, low field is advantageous in all ways for interventional work, when compared with higher fields such as $1.5 \mathrm{~T}$.

\section{SUMMARY}

A new, next-generation low-field MR design would benefit by the implementation of knowledge gained from the development of contemporary high-performance hardware used at higher fields. Image quality, regardless of field strength, is dependent upon main field homogeneity (achieved with a state-of-the-art magnet design), fast shielded gradient systems that minimize eddy currents, phased-array (multichannel) receiver coils, and advanced image reconstruction (including compressed sensing, for improved SNR/scan time) - the latter using inexpensive, readily available computation power. An advanced-design, substantially wider bore would add a new paradigm in patient comfort, in addition to its utility for patient monitoring, sedation, and interventional MR. Reduced acoustic noise would also improve patient comfort, as well as that of associated staff working in the MR environment.

Low-field MRI is also inherently more cost-effective owing to reduced magnet, RF power amplifier (RF transmitter), and siting costs. Installation and infrastructure (weight, size) requirements are significantly reduced. An advanced magnet design could also eliminate the need for helium refills and potentially the quench pipe. The reduction in unit cost, together with the simpler installation (and greater siting flexibility), has important implications for technology dissemination-both in developed economies and in underdeveloped areas ${ }^{14}$ - and access to care. This will further accelerate dissemination of MR technology to rural and less developed areas of the world, in analogy to the current revolution in ultrasound technology. ${ }^{33,34}$

And then there are specific imaging advantages to low field. The lower susceptibility leads to improved sequence performance and, in many anatomic areas, improved image quality. The intrinsically lower SAR adds sequence flexibility and diminishes the difficulties and concerns with metal implants and interventional techniques (the latter due to catheters, guidewires, and needles). The lower field makes possible advanced readout strategies, like spiral imaging, with increased SNR per measurement time. Signal-to-noise ratio-efficient long readout strategies can be used, with reduced image distortion and blurring.

\section{REFERENCES}

1. Attenberger UI, Biber S, Wichtmann BD. Technological advances of magnetic resonance imaging in today's healthcare environment. Invest Radiol. 2020;55: 531-542.

2. Wehrli FW. On the 2003 Nobel prize in medicine or physiology awarded to Paul C. Lauterbur and sir Peter Mansfield. Magn Reson Med. 2004;51:1-3.

3. Stalker F. The life-saving machine developed in Aberdeen. BBC; 2018. www.bbc.com/news/uk-scotland-north-east-orkney-shetland-44616538. Accessed November 21, 2019.

4. Crooks LE, Arakawa M, Hoenninger J, et al. Magnetic resonance imaging: effects of magnetic field strength. Radiology. 1984;151:127-133.
5. Edelman RR, Hahn PF, Buxton R, et al. Rapid MR imaging with suspended respiration: clinical application in the liver. Radiology. 1986;161:125-131.

6. Bradley WG Jr. Pros and cons of 3 tesla MRI. J Am Coll Radiol. 2008;5:871-878.

7. Ross JS. The high-field-strength curmudgeon. AJNR Am J Neuroradiol. 2004;25: 168-169.

8. Runge VM, Nitz WR, Schmeets SH, et al. Clinical 3 T Magnetic Resonance. New York: Thieme; 2007.

9. Schmitz BL, Aschoff AJ, Hoffmann MH, et al. Advantages and pitfalls in 3T MR brain imaging: a pictorial review. AJNR Am J Neuroradiol. 2005;26:2229-2237.

10. Lee DH, Vellet AD, Eliasziw M, et al. MR imaging field strength: prospective evaluation of the diagnostic accuracy of MR for diagnosis of multiple sclerosis at 0.5 and 1.5 T. Radiology. 1995;194:257-262.

11. Vellet AD, Lee DH, Munk PL, et al. Anterior cruciate ligament tear: prospective evaluation of diagnostic accuracy of middle- and high-field-strength MR imaging at 1.5 and 0.5 T. Radiology. 1995; 197:826-830.

12. Rutt BK, Lee DH. The impact of field strength on image quality in MRI. J Magn Reson Imaging. 1996;6:57-62.

13. Lewin JS, Laub G. Intracranial MR angiography: a direct comparison of three time-of-flight techniques. AJNR Am J Neuroradiol. 1991;12:1133-1139.

14. Geethanath S, Vaughan JT Jr. Accessible magnetic resonance imaging: a review. J Magn Reson Imaging. 2019;49:e65-e77.

15. Wald LL, McDaniel PC, Witzel T, et al. Low-cost and portable MRI [published online ahead of print October 12, 2019]. J Magn Reson Imaging.

16. DeBevits JJ, Munbodh R, Bageac D, et al. Gray matter nucleus hyperintensity after monthly triple-dose gadopentetate dimeglumine with long-term magnetic resonance imaging. Invest Radiol. 2020;55:629-635.

17. Panther A, Thevathasen G, Connell IRO, et al. A dedicated head-only MRI scanner for point-of-care imaging. Presented at: ISMRM 27th Annual Meeting \& Exhibition; May 11-16, 2019, Montréal, QC, Canada.

18. Campbell-Washburn AE, Ramasawmy R, Restivo MC, et al. Opportunities in interventional and diagnostic imaging by using high-performance low-fieldstrength MRI. Radiology. 2019;293:384-393.

19. Sarracanie M, Armstrong BD, Stockmann J, et al. High speed 3D overhauser-enhanced MRI using combined b-SSFP and compressed sensing. Magn Reson Med. 2014; 71:735-745.

20. Runge VM, Richter JK, Heverhagen JT. Motion in magnetic resonance: new paradigms for improved clinical diagnosis. Invest Radiol. 2019;54:383-395.

21. Kang HJ, Lee JM, Ahn SJ, et al. Clinical feasibility of gadoxetic acid-enhanced isotropic high-resolution 3-dimensional magnetic resonance cholangiography using an iterative denoising algorithm for evaluation of the biliary anatomy of living liver donors. Invest Radiol. 2019;54:103-109.

22. Klosowski J, Frahm J. Image denoising for real-time MRI. Magn Reson Med. 2017;77:1340-1352.

23. Chen G, Dong B, Zhang Y, et al. Denoising of diffusion MRI data via graph Framelet matching in X-q space. IEEE Trans Med Imaging. 2019;38:2838-2848.

24. Moelker A, Wielopolski PA, Pattynama PM. Relationship between magnetic field strength and magnetic-resonance-related acoustic noise levels. MAGMA. 2003;16: $52-55$.

25. Runge VM, Clanton JA, Price AC, et al. Dyke award. Evaluation of contrast-enhanced MR imaging in a brain-abscess model. AJNR Am J Neuroradiol. 1985;6:139-147.

26. Runge VM. Gd-DTPA: an I.V. contrast agent for clinical MRI. Int J Rad Appl Instrum B. 1988;15:37-44.

27. Runge VM, Bradley WG, Brant-Zawadzki MN, et al. Clinical safety and efficacy of gadoteridol: a study in 411 patients with suspected intracranial and spinal disease. Radiology. 1991;181:701-709.

28. Rohrer M, Bauer H, Mintorovitch J, et al. Comparison of magnetic properties of MRI contrast media solutions at different magnetic field strengths. Invest Radiol. 2005;40:715-724.

29. Shen Y, Goerner FL, Snyder C, et al. T1 relaxivities of gadolinium-based magnetic resonance contrast agents in human whole blood at 1.5, 3, and $7 \mathrm{~T}$. Invest Radiol. 2015;50:330-338.

30. Laurent S, Elst LV, Muller RN. Comparative study of the physicochemical properties of six clinical low molecular weight gadolinium contrast agents. Contrast Media Mol Imaging. 2006;1:128-137.

31. Desai NK, Runge VM. Contrast use at low field: a review. Top Magn Reson Imaging. 2003;14:360-364.

32. Yang K, Ganguli S, DeLorenzo MC, et al. Procedure-specific CT dose and utilization factors for CT-guided interventional procedures. Radiology. 2018;289:150-157.

33. Rothberg J. 12 Innovations that will change health care and medicine in the $2020 \mathrm{~s}$ An ultrasound in your pocket. Time. 2019.

34. Wang S, Hossack JA, Klibanov AL. From anatomy to functional and molecular biomarker imaging, and therapy: ultrasound is safe, ultrafast, portable, and inexpensive. Invest Radiol. 2020;55:559-572. 